\title{
Unique Bronchoscopy Findings in a Child with Severe Bronchiolitis Obliterans Following Overlapping Triggers
}

\author{
Dima R Ezmigna* \\ Department of pediatrics, University of Florida, USA
}

*Corresponding author: Dima R Ezmigna, Department of pediatrics, Pediatric

Pulmonary Division, University of Florida, USA.

Received Date: May 26, 2020

Published Date: June 12, 2020

\begin{abstract}
Acute pulmonary complications following Steven Johnson syndrome (SJS) are well recognized, in contrast, few cases have been reported describing chronic pulmonary complications [1]. We present a case of severe bronchiolitis obliterans (B0) with unique bronchoscopic findings following SJS overlapping with other triggers. Verbal consent was obtained from the caregiver prior to writing this case report.
\end{abstract}

Keywords: Bronchiolitis obliterans; Steven's Johnson syndrome; Mycoplasma pneumoniae

Abbreviations: (SJS): Steven Johnson syndrome; (BO): Bronchiolitis obliterans; (PE): Physical examination; (LUL): Left upper lobe; (FEV1): Forced expiratory volume at the first second; (FVC): Forced vital capacity; (CT): Computed tomography; (MP): Mycoplasma Pneumoniae

\section{Case Report}

An 11-year-old boy presented to the pediatric emergency department with cough, fever, shortness of breath and skin rash for several days duration. His physical examination (PE) showed an ill-appearing child with: Temperature: 39.3C, Heart Rate: 140 per minute, Respiratory Rate: 32 per minute, Blood Pressure: 130/80 and oxygen saturation of $93 \%$ on room air. Skin exam showed multiple erythematous and targetoid papules with central dusky necrosis involving the upper trunk and both extremities. Mucositis involving the upper and lower lips and buccal mucosa was noted. Eye exam with evidence of bilateral purulent conjunctivitis. Chest auscultation was positive for bilateral fine crackles. The rest of PE was unremarkable. His chest x-ray showed Peribronchial cuffing and thickening with mild left upper lobe (LUL) atelectasis. Due to the described skin involvement, SJS was considered and skin biopsy result was consistent with histopathologic changes of SJS. Mycoplasma Pneumonia was suspected, and serology was positive for IgA -specific antibodies. Respiratory viral panel and HSV PCR were negative. He was treated with five-day course of azithromycin, hydration, oxygen supplement and pain management. The patient was discharged home following stabilization. 4 weeks following discharge, he developed exertional dyspnea and was referred to pediatric pulmonology for consultation. His PE revealed diffuse bilateral expiratory wheezes with no signs of respiratory distress and his oxygen saturation was $98 \%$ on room air. Skin exam showed post inflammatory hyperpigmented lesions involving the trunk and extremities. The rest of the PE was unremarkable. He was treated with inhaled bronchodilator (albuterol) and systemic steroids for 5 days and upon follow up he reported partial response with continued wheezing and dyspnea. Follow up CXR showed worsening LUL atelectasis with left-sided mediastinal shift. Spirometry showed moderately-severe airway obstruction that is not reversible following bronchodilator therapy with forced 
expiratory volume at the first second (FEV1) at $46 \%$ predicted and forced vital capacity (FVC) of 78\% predicted and a ratio of FEV1/FVC of 52. This prompted obtaining urgent computed tomography (CT) of chest (Figure 1) which showed mosaic attenuation of the right lung, LUL atelectasis and a small left lung with vascular attenuation and a mediastinal shift to the left side; findings suggestive of BO. Flexible bronchoscopy revealed inflamed mucosa, increased mucous plugs in the LUL with difficulty to pass the scope through the LUL bronchus due to scarring and complete obliteration (Figure 2). Bronchoalveolar lavage analysis with dominant neutrophils (71\%) and bacterial culture was positive for Haemophilus Influenza. Transbronchial biopsy showed nonspecific mild chronic inflammation. Despite insufficient transbronchial biopsy results, the patient was diagnosed with BO based on the clinical picture and radiology findings. He was treated with Methylprednisolone pulse therapy (10 mg/kg/dose) daily for 3 days repeated monthly for 3 courses with minimal improvement on spirometry. Ventilation/ perfusion scan was obtained and showed ventilation of $91 \%$ on the right lung and $9 \%$ on the left with matched perfusion of $87 \%$ on the right lung and $13 \%$ on the left lung. Upon follow up 3 months later, chest CT revealed worsening of the above findings with complete collapse of the left lung and herniation of the hyperexpanded right lung to the left hemi-mediastinum. Spirometry findings became consistent with a restrictive rather than an obstructive pathology. Despite radiologic worsening, the patient remained stable on room air. FEV1 remained at $46 \%$.
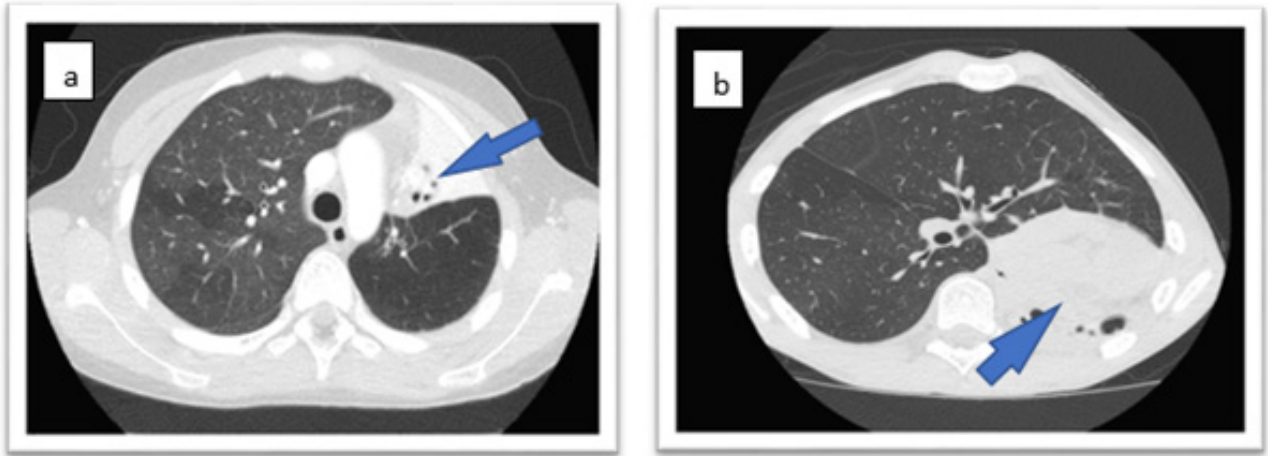

Figure 1: (a) CT scan of the chest showing mosaic attenuation, left upper lobe atelectasis (arrow) and herniation of the right lung into the left. (b) Follow up 3 months later shows complete collapse of the left lung and herniation of the right lung to the left hemithorax (arrow).

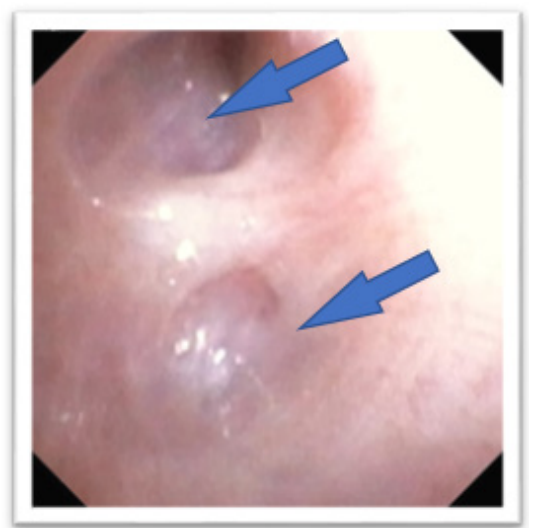

Figure 2: Bronchoscopy image showing complete obliteration and scarring of the left upper lobe bronchus.

\section{Discussion}

BO or constrictive bronchiolitis is a chronic obstructive pulmonary disease caused by immune and nonimmune - mediated triggers resulting in airway inflammation and epithelial cell injury leading to increased fibroblast and myofibroblast activity during the healing process [1]. The latter results in the formation of scar tissue obliterating the small airways - the bronchioles. Diagnosis of $\mathrm{BO}$ in children can be made with confidence based on the clinical picture and associated radiological findings in addition to fixed pulmonary obstructive disease [2]. Large airways are usually spared, making this case unique in describing proximal involvement of the bronchial tree with fibrosis and scarring captured on flexible bronchoscopy images, in this case involving the LUL bronchus.

In this report of severe $\mathrm{BO}$, another distinctive finding is the overlap of 2 pathologic triggers, both of which have been separately reported to cause BO; Stevens Johnson Syndrome (SJS) and Mycoplasma Pneumoniae (MP) infection. MP is currently a wellrecognized cause of post-infectious $\mathrm{BO}$ in the pediatric and adult population. Our patient was diagnosed with MP 4-6 weeks prior to the development of chronic wheezing and dyspnea, however, this 
coincided with the occurrence of SJS; an immunologic trigger that is believed to have simultaneously contributed to the pathogenesis of BO and abnormal pulmonary function subsequently. SJS is a severe mucocutaneous reaction that is commonly triggered by medications including sulfonamides, anticonvulsants, and nonsteroidal anti-inflammatory drugs, as well as infectious etiologies especially in the pediatric population. In severe cases of SJS, multisystemic involvement is a sequela, including the pulmonary system. In the acute phase, pulmonary involvement following SJS is commonly reported and can affect up to $40 \%$ of patients 3 with pneumonitis, acute respiratory distress, and respiratory failure requiring mechanical ventilation. In contrast, chronic pulmonary complications are less common, and are being recognized over time through case reports and case series $[1,3,4]$ including $\mathrm{BO}$ and abnormal lung function [5]. The severity of this case and the progressive findings on chest CT suggest continued active airway inflammation for several weeks despite immune suppression by Methylprednisolone pulse therapy. This led to the development of profound scarring involving the left lower lobe leading to the complete collapse of the left lung, which contributed to the restrictive pattern demonstrated on spirometry on a later follow up. Long term Azithromycin therapy, another agent described in the management of BO due to its immune-modulatory effect (although with variable outcomes [6]), was avoided in this case given the possibility that it may have contributed to the development/worsening of SJS.

\section{Summary}

This is a unique case of BO with severe large airway involvement secondary to overlapping triggers; MP and SJS. The development of respiratory symptoms following SJS should prompt early evaluation and management by a pediatric pulmonologist to prevent serious irreversible lung damage. Response to current available therapies is not promising, and lung transplant is the ultimate treatment in severe cases [2].

\section{Acknowledgment}

None.

\section{Conflict of Interest}

No conflict of interest.

\section{References}

1. Sugino K, Hebisawa A, Uekusa T, Hatanaka K, Abe H, et al. (2013) Bronchiolitis obliterans associated with Stevens-Johnson Syndrome: histopathological bronchial reconstruction of the whole lung and immunohistochemical study. Diagn Pathol 8: 134.

2. Moonnumakal Siby P, Fan Leland L (2008) Bronchiolitis obliterans in children. Curr Opin Pediatr 20(3): 272-278.

3. Hyungchul P, Young BK, Hyouk SK, Chae ML (2015) Bronchiolitis Obliterans Associated with Stevens-Johnson Syndrome: A Case Report. Yonsei Med J 56(2): 578-581.

4. Lee HY, Walsh SA, Creamer D (2017) Long-term complications of Stevens-Johnson syndrome/toxic epidermal necrolysis (SJS/TEN): the spectrum of chronic problems in patients who survive an episode of SJS/TEN necessitates multidisciplinary follow-up. Br J Dermatol 177(4): 924-935.

5. Duong TA, de Prost N, Housz Oro SI, Carrié AS, Zerah F, et al. (2015) Stevens-Johnson syndrome and toxic epidermal necrolysis: follow-up of pulmonary function after remission. Br J Dermatol 172(2): 400-405.

6. Hakim A, Cooke KR, Pavletic SZ, Khalid M, Williams KM, et al. (2019) Diagnosis and treatment of bronchiolitis obliterans syndrome accessible universally. Bone Marrow Transplant 54(3): 383-392. 